\title{
Evaluación del riesgo de síndrome de apnea obstructiva del sueño y somnolencia diurna utilizando el cuestionario de Berlín y las escalas Sleep Apnea Clinical Score y Epworth en pacientes con ronquido habitual atendidos en la consulta ambulatoria
}

\author{
CÉSAR CHÁVEZ-GONZÁLES* y ALONSO SOTO T.****
}

\begin{abstract}
Risk assessment for Obstructive Sleep Apnea Syndrome and daytime sleepiness using Berlin questionnaire, and SACS and Epworth scales in outpatients with snoring attending consultation
\end{abstract}

Objectives: To determine the frequency of risk for the obstructive sleep apnea syndrome (OSA) through clinical predictors: Berlin Questionnaire, Sleep Apnea Clinical Score (SACS) and the degree of daytime sleepiness measured by Epworth scale. Material and Methods: A cross-sectional study was conducted among patients who reported snoring seen by pulmonology in the outpatient clinic between January and March 2014. Frequency of OSA was calculated according to the three clinical prediction rules. We also determine the characteristic associated with a high risk of OSA according to each prediction rule. Results: We recruited 230 participants, $56.5 \%$ were male, with a mean age of $50 \pm 12$ years. The risk of moderate or high risk for OSA was $61.8 \%, 66.9 \%$ and $62.6 \%$ according to Epworth somnolence scale, SACS and Berlin questionnaire, respectively. Neck circumference, abdominal circumference and body mass index were the characteristics more consistently associated with OSA risk. Correlation between SACS and Berlin prediction rules was 0.55, between SACS and Epworth scale was 0.22 and between Berlin and Epworth scale was 0.35 ( $p<0.001$ for all comparisons). Conclusions: There is a high risk for OSA among snoring patients attending respiratory outpatient clinic. The correlation between prediction rules evaluated was lower than expected. Larger studies in general populations using polysomnography as a reference standard are needed to clarify the diagnostic and prognostic value of OSA prediction rules.

Key words: Obstructive sleep apnea; daytime sleepiness; Berlin questionnaire; Epworth scale; sleep apnea clinical score.

\section{Resumen}

Objetivos: Determinar la frecuencia de riesgo del Síndrome de Apnea Obstructiva del Sueño (SAOS) en pacientes atendidos en el consultorio externo de neumología de un hospital de referencia en Lima a través de los Cuestionarios de Berlín, Sleep Apnea Clinical Score (SACS) y la escala de Epworth. Métodos: Se realizó un estudio transversal en pacientes atendidos en los consultorios externos de neumología reclutando durante el periodo de enero a marzo de 2014 en forma prospectiva a aquellos pacientes que referían presentar ronquido habitual. Se determinó la frecuencia de riesgo de SAOS de acuerdo a cada una de las escalas evaluadas y sus factores asociados. Resultados: Se estudiaron 230 sujetos, 56,5\% eran varones y el 43,5\% mujeres, con una edad media de $50 \pm 12$ años. El

CC y AS participaron en la concepción del estudio, análisis, elaboración del manuscrito y aprobación de su versión final. CC participó en el reclutamiento de participantes. Ambos autores se hacen responsables de los contenidos del artículo.

* Hospital Nacional Hipólito Unánue. Lima, Perú.

** Escuela de Medicina. Universidad Peruana de Ciencias Aplicadas. Lima, Perú. 
$61,8 \%, 66,9 \%$ y 62,6\% tenían riesgo moderado o alto para SAOS de acuerdo a las escalas de Epworth, SACS y Berlin respectivamente. El perímetro de cuello, circunferencia abdominal e índice de masa corporal fueron las características asociadas de manera más consistente con el riesgo de SAOS. La correlación entre las escalas de SACS y Berlin fue de 0,55, entre las escalas SACS y Epworth de 0,22 y entre Berlin y Epworth de 0,35 ( $p<0,001$ para todas las comparaciones). Conclusiones: El riesgo de SAOS y somnolencia diurna es elevado en pacientes roncadores atendidos en la consulta externa de neumología. Las escalas evaluadas presentan una correlación menor que lo esperada. Se requieren estudios a mayor escala y en población general que comparen el valor diagnóstico y pronóstico de estas escalas utilizando la polisomnografía como estándar de referencia.

Palabras clave: Apnea obstructiva del sueño; somnolencia diurna; cuestionario de Berlín; escala de Epworth; sleep apnea clinical score.

\section{Introducción}

El Síndrome de Apnea Obstructiva del Sueño (SAOS) es definido por la Academia Americana de Medicina del Sueño (AAMS) como una enfermedad caracterizada por episodios repetitivos de obstrucción total (apnea) o parcial (hipopnea) de la vía aérea superior durante el sueño. Estos eventos ocasionan disminución de la saturación de oxígeno en la sangre y suelen terminar en un breve despertar ${ }^{1}$. El SAOS representa un problema de salud pública por su elevada prevalencia y los graves daños a la salud que ocasiona, particularmente en escenarios de recursos limitados con escasa disponibilidad de procedimientos diagnósticos y tratamiento oportunos ${ }^{1}$. La mayoría de los estudios con base poblacional describen prevalencias de SAOS de alrededor del 3\%, la cual aumenta hasta un $25 \%$ en roncadores habituales ${ }^{2-5}$. Ello es especialmente relevante considerando que la frecuencia de reporte de ronquido llega hasta el $60 \%$ en población latina ${ }^{6}$.

En el Perú existen pocos datos epidemiológicos de frecuencia, prevalencia o incidencia de esta entidad. El determinar qué individuos están en riesgo de presentar este síndrome es importante ya que el SAOS es un factor de riesgo para desarrollar hipertensión arterial, eventos cerebrovasculares o infarto agudo de miocardio e hipertrofia ventricular izquierda, debido a la hipoxemia, hipercapnia y obstrucción de las vías aéreas resultantes ${ }^{7,8}$.

El diagnóstico de esta condición es difícil y la historia clínica realizada incluso por un especialista en sueño sólo alcanza una sensibilidad de $64 \%{ }^{9}$. Actualmente, la polisomnografía constituye el estándar de referencia para establecer el diagnóstico de SAOS. Sin embargo, consume mucho tiempo, no es universalmente accesible y su costo es muy elevado ${ }^{10,11}$, por lo cual es importante contar con herramientas clínicas simples que permitan identificar a aquellas personas con mayor riesgo de presentar SAOS. Las reglas de predicción clínica pueden ser útiles para definir aquellos pacientes en quienes se debería realizar un estudio polisomnográfico o un estudio simplificado (poligrafía respiratoria e incluso oximetrías nocturnas), para establecer un diagnóstico definitivo de SAOS. Una de las reglas de predicción clínica más utilizadas es el sleep apnea clinical score (SACS). El SACS tiene una sensibilidad del $90 \%$ y especificidad del $63 \%$ en la pesquisa de SAOS y se basa en la medición del perímetro de cuello, hipertensión arterial sistémica, ronquido habitual y reporte de apneas presenciadas por el compañero de habitación ${ }^{12}$. El cuestionario de Berlín (CB) es otro método sencillo y validado para predecir el riesgo de SAOS en la población en general y en pacientes con enfermedades cardiovasculares ${ }^{13}$. Este cuestionario ${ }^{14}$ examina la presencia de síntomas persistentes de ronquidos y apneas; excesiva somnolencia diurna o al conducir vehículos o ambos; e historia de hipertensión arterial o índice de masa corporal superior a 30 $\mathrm{kg} / \mathrm{m}^{2}$. Finalmente, la escala de Epworth ${ }^{15}$, basada en preguntas simples relacionadas a la tendencia de quedarse dormido en situaciones cotidianas, cuantifica el grado de somnolencia diurna, elemento de gran importancia para la identificación de pacientes con riesgo de Apnea Obstructiva del Sueño. Esta escala ha sido utilizada en estudios poblacionales ${ }^{6} \mathrm{y}$ recomendada para la pesquisa de pacientes con SAOS en atención primaria ${ }^{16}$.

El objetivo del presente estudio fue determinar la frecuencia del riesgo del Síndrome de Apnea Obstructiva del Sueño evaluado a través del cuestionario de Berlín, la escala SACS y el grado de somnolencia diurna medido a través de la escala Epworth en pacientes roncadores atendidos en el consultorio externo de un hospital peruano de referencia. Como objetivos secundarios se evaluó la asociación de diferentes parámetros clínicos y antropométricos con la presencia de SAOS y la correlación entre las tres escalas. 


\section{Material y Métodos}

Se realizó un estudio transversal analítico que incluyó pacientes mayores de 18 años atendidos por cualquier síntoma respiratorio en los consultorios externos de Neumología del Hospital Nacional Hipólito Unanue durante el período de enero a marzo de 2014, quienes referían presentar ronquidos de manera habitual. En base a una frecuencia esperada del $50 \%$ de riesgo de SAOS o somnolencia entre roncadores, con una precisión del $5 \%$ y una significancia de 0,05 , el tamaño muestral calculado fue de 227 pacientes.

Durante el período referido, fueron atendidos 1.108 pacientes en la consulta externa, de los cuales 249 referían presentar ronquido habitual, 10 no desearon participar del estudio y 9 formatos de recolección presentaron datos incompletos (porcentaje de pérdidas: 3,7\%), finalmente se enrolaron 230 participantes, los cuales firmaron el consentimiento informado autorizado por el Comité de Ética del hospital.

Se realizó el registro de una ficha a cargo de uno de los investigadores (CCG) conteniendo variables sociodemográficas y clínicas incluyendo edad, género, horas de sueño, peso, talla, circunferencia de cuello, circunferencia abdominal e índice de masa corporal (IMC). También se valoró el tipo de cavidad oral a través de la Escala de Mallampati, y el grado de hipertrofia amigdalina identificado mediante examen clínico. Se aplicaron el cuestionario de Berlín, la escala de somnolencia de Epworth y el SACS a todos los participantes. La escala de Epworth evalúa el grado de somnolencia diurna, consiste en la obtención de un puntaje de 0 a 3 para cada una de ocho preguntas en relación a la tendencia de quedarse dormido en situaciones cotidianas. $\mathrm{Si}$ el puntaje es superior a nueve se considera somnolencia y si supera más de 12 se define somnolencia diurna excesiva. Se utilizó la validación peruana desarrollada por Rosales ${ }^{17}$.

El cuestionario de Berlín evalúa el riesgo de SAOS en base a las respuestas a tres categorías: 1) Síntomas persistentes de ronquidos y apneas; 2) Síntomas persistentes de excesiva somnolencia diurna, conducir con sueño o ambos y, 3) Historia de hipertensión arterial o IMC superior a $30 \mathrm{~kg} /$ $\mathrm{m}^{2}$. Se considera alto riesgo para SAOS si presentan dos o más categorías. Se utilizó la versión validada en Colombia por Polanía-Dussan et al ${ }^{18}$.

El puntaje SACS se basa en la medición del perímetro de cuello en centímetros (con el paciente sentado, cuello en posición neutra y a nivel de la membrana cricotiroidea), a esta medida se suman 4 puntos si el paciente padece hipertensión arterial sistémica, 3 en presencia de ronquido habitual (casi todos los días) y 3 en caso que se reporten apneas presenciadas por el compañero de habitación (casi todos los días). Se estima el riesgo de padecer la enfermedad de acuerdo al puntaje obtenido, considerando el riesgo como bajo en casos con puntaciones menores a 43 , riesgo moderado entre 43 y 48 y riesgo alto en casos con más de 48 puntos $^{19}$.

\section{Análisis estadístico}

Se utilizó el método de Kolmogorov-Smirnov para determinar la distribución normal de las variables. Para las comparaciones se utilizaron las pruebas t de Student, ANOVA, Chi cuadrado de Pearson y test exacto de Fisher. Las correlaciones entre los puntajes se evaluaron utilizando los coeficientes de correlación de Pearson y Spearman. El análisis de datos se realizó con el programa Stata versión 11.0.

\section{Resultados}

Se incluyeron 230 sujetos, la media de edad de los pacientes fue $50 \pm 12$ años correspondiendo $130(56,5 \%)$ al género masculino. El 28\% de los pacientes tenía hipertensión arterial, el 43\% presentaron un índice de Mallampati de III o IV, el IMC promedio fue $33,4 \mathrm{~kg} / \mathrm{m}^{2}$ y el $64 \%$ de los pacientes eran obesos. La media de saturación arterial de oxígeno fue de 97,1\%. Las Tablas 1, 2 y 3 muestran la frecuencia de las variables clínicas asociadas al riesgo de somnolencia diurna excesiva y/o riesgo de SAOS.

La evaluación mediante el cuestionario de Berlín evidenció una frecuencia de pacientes en riesgo de SAOS de 62,6\%. La evaluación mediante la escala de Epworth mostró la presencia de somnolencia en $61,8 \%$ (incluyendo un $32,2 \%$ con somnolencia excesiva), mientras que la escala SACS encontró riesgo bajo en $33,0 \%$, riesgo moderado en $41,7 \%$ y riesgo alto en $25,2 \%$. Las tres escalas mostraron hallazgos consistentes con respecto a la frecuencia de trastornos respiratorios del sueño con rangos bastante comparables entre 62 y $66 \%$. En cuanto la escala de Berlín, el riesgo de SAOS se asoció significativamente a la escala de Mallampati, hipertrofia amigdalina, perímetro de cuello y perímetro abdominal.

Al evaluar el riesgo mediante la escala de Epworth se encontró como factores asociados a riesgo de SAOS el número de horas de sueño, la escala de Mallampati, la hipertrofia amigdalina, el perímetro de cuello, el perímetro abdominal, la saturación de oxígeno y un IMC elevado. La 
Tabla 1. Comparación de variables clínicas de acuerdo a riesgo de SAOS medido mediante la escala de Berlín en pacientes atendidos en consultorio externo de neumología. Lima, Perú. 2014

\begin{tabular}{|c|c|c|c|c|c|c|}
\hline & \multicolumn{2}{|c|}{$\begin{array}{c}\text { Total } \\
(n=230)\end{array}$} & \multicolumn{2}{|c|}{$\begin{array}{l}\text { Riesgo SAOS } \\
\quad(n=144)\end{array}$} & $\begin{array}{c}\text { No Riesgo SAOS } \\
(n=80)\end{array}$ & Valor de $p$ \\
\hline Edad (años) & \multicolumn{2}{|c|}{$49,76(11,8)$} & \multicolumn{2}{|c|}{$50,89(10,48)$} & $47,86(13,57)$ & $0,06^{*}$ \\
\hline Género masculino & 130 & $(56,5 \%)$ & 78 & $(54,2 \%)$ & $52 \quad(60,5 \%)$ & $0,35 * *$ \\
\hline Horas de sueño & & & & & & $0,25 * * *$ \\
\hline Menor de $7 \mathrm{~h}$ & 149 & $(64,84 \%)$ & 95 & $(65,0 \%)$ & $54 \quad(62,8 \%)$ & \\
\hline 7 a $9 \mathrm{~h}$ & 77 & $(33,5 \%)$ & 45 & $(31,3 \%)$ & $32 \quad(37,2 \%)$ & \\
\hline Mayor a $9 \mathrm{~h}$ & 4 & $(1,7 \%)$ & 4 & $(2,8 \%)$ & $(0 \%)$ & \\
\hline Mallampati & & & & & & $0,001 * *$ \\
\hline Clase I & 29 & $(12,6 \%)$ & 18 & $(12,5 \%)$ & $11 \quad(12,8 \%)$ & \\
\hline Clase II & 103 & $(44,8 \%)$ & 53 & $(36,8 \%)$ & $50 \quad(58,1 \%)$ & \\
\hline Clase III & 72 & $(31,3 \%)$ & 58 & $(40,3 \%)$ & $14 \quad(16,3 \%)$ & \\
\hline Clase IV & 26 & $(11,3 \%)$ & 15 & $(10,4 \%)$ & $11 \quad(12,8 \%)$ & \\
\hline Hipertrofia amigdalina & & & & & & $<0,01 * * *$ \\
\hline Grado 0 & 45 & $(19,6 \%)$ & 31 & $(21,5 \%)$ & $14 \quad(16,3 \%)$ & \\
\hline Grado 1 & 134 & $(58,3 \%)$ & 71 & $(49,3 \%)$ & $63 \quad(73,3 \%)$ & \\
\hline Grado 2 & 47 & $(20,4 \%)$ & 38 & $(26,4 \%)$ & $(10,5 \%)$ & \\
\hline Grado 3 & 2 & $(0,9 \%)$ & 2 & $(1,4 \%)$ & $(0 \%)$ & \\
\hline Grado 4 & 2 & $(0,9 \%)$ & 2 & $(1,4 \%)$ & $(0 \%)$ & \\
\hline Perímetro de cuello (cm) & 40,6 & $(4,03)$ & 41,29 & $(4,26)$ & $39,45(3,32)$ & $<0,001^{*}$ \\
\hline Perímetro abdominal $(\mathrm{cm})$ & 109,5 & $5(14,0)$ & 112,8 & $(14,66)$ & $104,0(10,81)$ & $<0,001^{*}$ \\
\hline Saturación de oxígeno (\%) & 97,0 & $(1,36)$ & $97,0^{-}$ & $(1,48)$ & $97,11(1,31)$ & $0,80^{*}$ \\
\hline Frecuencia cardiaca (lat/min) & 75,9 & $7(10,10)$ & 75,56 & $(10,31)$ & $76,64(9,76)$ & $0,43 *$ \\
\hline
\end{tabular}

SAOS: Síndrome de apnea obstructiva del sueño. *t de Student; **Chi cuadrado; ***prueba exacta de Fisher.

escala SACS encontró como factores asociados el género, perímetro abdominal, saturación de oxígeno y obesidad. El perímetro de cuello, perímetro abdominal e índice de masa corporal fueron las características asociadas al riesgo de SAOS de manera más consistente al evaluar las tres escalas (Tabla 4).

El coeficiente de correlación entre las escalas Epworth y SACS fue de $0,22(\mathrm{p}<0,001)$, entre Epworth y Berlín $0,35(\mathrm{p}<0,001)$ y entre Berlín y SACS de 0,55 ( $\mathrm{p}<0,001)$ (Figuras 1 a 3 ).

\section{Discusión}

Nuestro estudio encontró de manera consistente un alto riesgo de SAOS en pacientes con ronquido habitual utilizando tres reglas de predicción clínica distintas, siendo el índice de masa corporal (IMC), perímetro de cuello (PC) y el perímetro abdominal (PA), las variables clínicas más consistentemente asociadas con el riesgo de SAOS. A pesar que el porcentaje de pacientes en riesgo de SAOS fue bastante similar (alrededor del $60 \%$ ), la correlación entre las tres escalas evaluadas fue menor a lo esperado, particularmente entre las escalas SACS y de Epworth. Ello es importante, pues a pesar de usarse de manera casi intercambiable en la práctica clínica, estas tres escalas representan conceptos diferentes. De hecho, el cuestionario de Berlín y la escala SACS buscan específicamente medir el riesgo de SAOS mientras que la escala de Epworth evalúa la presencia de somnolencia excesiva diurna, el que a su vez es un marcador de riesgo de SAOS.

Aunque nuestros hallazgos encuentran una alta proporción de pacientes con riesgo de SAOS utilizando los tres instrumentos, no es posible saber a ciencia cierta cuál de las tres escalas podría ser más útil desde el punto de vista clínico para poder tamizar a los pacientes de manera más adecuada para evaluar el riesgo de SAOS al no haberse podido realizar el estándar de referencia (es decir, la polisomnografía) para el diagnóstico de esta condición. 
Tabla 2. Comparación de variables clínicas de acuerdo a riesgo de SAOS medido mediante la escala de Epworth en pacientes atendidos en consultorio externo de neumología. Lima, Perú. 2014

\begin{tabular}{|c|c|c|c|c|c|}
\hline & $\begin{array}{c}\text { Total } \\
(\mathrm{n}=\mathbf{2 3 0})\end{array}$ & $\begin{array}{c}\text { No refiere } \\
\text { somnolencia } \\
\quad(\mathbf{n}=\mathbf{8 8})\end{array}$ & $\begin{array}{l}\text { Somnolencia } \\
\qquad(n=68)\end{array}$ & $\begin{array}{c}\text { Somnolencia } \\
\text { excesiva diur- } \\
\text { na }(n=74)\end{array}$ & Valor de p \\
\hline Edad (años) & $49,76(11,8)$ & $49,8 \quad(11,7)$ & $48,5(12,9)$ & $50,9(10,8)$ & $0,47^{*}$ \\
\hline Género masculino & $(56,5 \%)$ & $51 \quad(58,0 \%)$ & $39 \quad(57,4 \%)$ & $(54,1 \%)$ & $0,87 * *$ \\
\hline Hipertensión arterial & $(72,2 \%)$ & $(75 \%)$ & $47 \quad(69 \%)$ & $53 \quad(71,6 \%)$ & $0,71 * *$ \\
\hline Horas de sueño & & & & & $0,04 * * *$ \\
\hline Menor de $7 \mathrm{~h}$ & $(64,84 \%)$ & $(56,8 \%)$ & $45 \quad(66,2 \%)$ & $(73,0 \%)$ & \\
\hline 7 a $9 \mathrm{~h}$ & $(33,5 \%)$ & $(43,2 \%)$ & $23 \quad(33,8 \%)$ & $16 \quad(21,6 \%)$ & \\
\hline Mayor a $9 \mathrm{~h}$ & $(1,7 \%)$ & $(0 \%)$ & $(0 \%)$ & $(5,4 \%)$ & \\
\hline Mallampati & & & & & $<0,01 * *$ \\
\hline Clase I & $(12,6 \%)$ & $(12,5 \%)$ & $(11,8 \%)$ & $(13,5 \%)$ & \\
\hline Clase II & $(44,8 \%)$ & $(38,6 \%)$ & $37 \quad(54,4 \%)$ & $(43,2 \%)$ & \\
\hline Clase III & $(31,3 \%)$ & $(28,4 \%)$ & $23 \quad(33,8 \%)$ & $(32,4 \%)$ & \\
\hline Clase IV & $(11,3 \%)$ & $(20,5 \%)$ & $(0 \%)$ & $(10,8 \%)$ & \\
\hline Hipertrofia amigdalina & & & & & $<0,01 * * *$ \\
\hline Grado 0 & $(19,6 \%)$ & $(21,6 \%)$ & $16 \quad(23,5 \%)$ & $(13,5 \%)$ & \\
\hline Grado 1 & $(58,3 \%)$ & $(61,4 \%)$ & $46 \quad(67,65 \%)$ & $(45,9 \%)$ & \\
\hline Grado 2 & $(20,4 \%)$ & $(17,1 \%)$ & $(8,8 \%)$ & $26 \quad(35,1 \%)$ & \\
\hline Grado 3 & $(0,9 \%)$ & $(0 \%)$ & $(0 \%)$ & $(2,7 \%)$ & \\
\hline Grado 4 & $(0,9 \%)$ & $(0 \%)$ & $(0 \%)$ & $(2,7 \%)$ & \\
\hline Perímetro de cuello (cm) & $40,6 \quad(4,03 \%)$ & $40,7 \quad(4,2 \%)$ & $39,8 \quad(3,2 \%)$ & $41,2 \quad(4,4 \%)$ & $<0,001 *$ \\
\hline Perímetro abdominal (cm) & $109,55(14,0)$ & $110,2(15,9)$ & $105,9(10,4)$ & $112,1(13,9)$ & $<0,001^{*}$ \\
\hline Saturación de oxígeno (\%) & $97,1 \quad(1,36)$ & $97,2 \quad(1,0)$ & $97,3 \quad(1,3)$ & $96,8 \quad(1,7)$ & $0,03^{*}$ \\
\hline Frecuencia cardiaca (lat/min) & $75,97(10,10)$ & $77,6 \quad(11,3)$ & $75,4(10,1)$ & $74,4 \quad(8,3)$ & $0,12 *$ \\
\hline $\mathrm{IMC}\left(\mathrm{kg} / \mathrm{m}^{2}\right)$ & $33,38 \quad(7,07)$ & $33,12(6,48)$ & $30,85(6,09)$ & $35,40(7,92)$ & $<0,01$ \\
\hline Obesidad & $(49,13 \%)$ & $46 \quad(52,3 \%)$ & $26 \quad(38,24 \%)$ & $41 \quad(55,4 \%)$ & \\
\hline Obesidad mórbida & $(14,8 \%)$ & $(9,1 \%)$ & $9 \quad(13,2 \%)$ & $(23,0 \%)$ & $<0,01 * *$ \\
\hline
\end{tabular}

SAOS: Síndrome de apnea obstructiva del sueño.*Análisis de Varianza. **Chi cuadrado. ***prueba exacta de Fisher.

Las tres reglas de predicción clínica evaluadas son consideradas adecuadas para tamizar a los individuos con riesgo de presentar SAOS de acuerdo a varios estudios. Por ejemplo, se ha encontrado que el SACS puede tener una sensibilidad de hasta el $96 \%$ para predecir un índice de apnea-hipopnea (IAH) compatible con SAOS $^{19}$. De manera similar, el cuestionario de Berlín ha mostrado una sensibilidad y valor predictivo de hasta 86 y $89 \%$ respectivamente ${ }^{13}$. La escala de Epworth por otro lado ha mostrado resultados comparables al cuestionario de Berlín, particularmente para la predicción de SAOS grave $^{20}$. Aunque todos estos estudios muestran un buen desempeño de estos sistemas de puntaje, la mayoría de ellos no han evaluado la correlación entre los mismos.

Entre otras variables, el riesgo de SAOS también está relacionado con la edad, siendo más frecuente después de los 40 años ${ }^{6,12}$. En nuestro estudio se encontró una edad promedio de 50 años, aunque no se evidenció asociación entre esta variable y el riesgo de SAOS. Por otro lado, el SAOS es un trastorno asociado principalmente al sobrepeso y la obesidad. Nuestro estudio encontró que un mayor IMC fue consistentemente asociado a la presencia de SAOS. Se ha visto que pacientes con un IMC mayor de 40 $\mathrm{kg} / \mathrm{m}^{2}$ incrementan hasta en $10 \%$ la frecuencia de riesgo ${ }^{21}$. Palla y cols., encontraron en centros de cirugía bariátrica una frecuencia de riesgo de hasta $60 \%{ }^{22}$. La circunferencia de cuello es un 
Tabla 3. Comparación de variables clínicas de acuerdo a riesgo de SAOS medido mediante la escala SACS en pacientes atendidos en consultorio externo de neumología. Lima, Perú. 2014

\begin{tabular}{|c|c|c|c|c|c|c|c|c|c|}
\hline & \multicolumn{2}{|c|}{$\begin{array}{c}\text { Total } \\
(\mathbf{n}=\mathbf{2 3 0})\end{array}$} & \multicolumn{2}{|c|}{$\begin{array}{l}\text { Riesgo bajo } \\
(\mathrm{n}=76)\end{array}$} & \multicolumn{2}{|c|}{$\begin{array}{c}\text { Riesgo moderado } \\
\quad(\mathrm{n}=96)\end{array}$} & \multicolumn{2}{|c|}{$\begin{array}{l}\text { Riesgo alto } \\
\quad(\mathbf{n}=\mathbf{5 8})\end{array}$} & \multirow{3}{*}{$\begin{array}{c}\begin{array}{c}\text { Valor } \\
\text { de p }\end{array} \\
0,06^{*} \\
<0,001^{* *}\end{array}$} \\
\hline Edad (años) & \multicolumn{2}{|c|}{$49,76(11,80)$} & \multicolumn{2}{|c|}{$47,18(11,39)$} & \multicolumn{2}{|c|}{$50,71(12,26)$} & \multicolumn{2}{|c|}{$51,58(11,12)$} & \\
\hline Género masculino & 130 & $(56,5 \%)$ & 25 & $(32,9 \%)$ & 60 & $(62,5 \%)$ & 45 & $(77,6 \%)$ & \\
\hline Horas de sueño & & & & & & & & & $0,06^{* * *}$ \\
\hline Menor de $7 \mathrm{~h}$ & 149 & $(64,84 \%)$ & 52 & $(68,4 \%)$ & 54 & $(56,3 \%)$ & 43 & $(74,1 \%)$ & \\
\hline 7 a 9 horas & 77 & $(33,5 \%)$ & 24 & $(31,6 \%)$ & 38 & $(39,6 \%)$ & 15 & $(25,9 \%)$ & \\
\hline Mayor a $9 \mathrm{~h}$ & 4 & $(1,7 \%)$ & 0 & $(0 \%)$ & 4 & $(4,2 \%)$ & 0 & $(0 \%)$ & \\
\hline Mallampati & & & & & & & & & $0,19 * *$ \\
\hline Clase I & 29 & $(12,6 \%)$ & 10 & $(13,2 \%)$ & 9 & $(9,4 \%)$ & 10 & $(17,2 \%)$ & \\
\hline Clase II & 103 & $(44,8 \%)$ & 41 & $(54,0 \%)$ & 44 & $(45,8 \%)$ & 18 & $(31,0 \%)$ & \\
\hline Clase III & 72 & $(31,3 \%)$ & 18 & $(23,7 \%)$ & 32 & $(33,3 \%)$ & 22 & $(37,9 \%)$ & \\
\hline Clase IV & 26 & $(11,3 \%)$ & 7 & $(9,2 \%)$ & 11 & $(11,5 \%)$ & 8 & $(13,8 \%)$ & \\
\hline Hipertrofia amigdalina & & & & & & & & & $0,13 * * *$ \\
\hline Grado 0 & 45 & $(19,6 \%)$ & 14 & $(18,4 \%)$ & 16 & $(16,7 \%)$ & 15 & $(25,9 \%)$ & \\
\hline Grado 1 & 134 & $(58,3 \%)$ & 50 & $(65,8 \%)$ & 57 & $(59,4 \%)$ & 27 & $(46,6 \%)$ & \\
\hline Grado 2 & 47 & $(20,4 \%)$ & 12 & $(15,8)$ & 21 & $(21,9 \%)$ & 14 & $(24,1 \%)$ & \\
\hline Grado 3 & 2 & $(0,9 \%)$ & 0 & $(0 \%)$ & 2 & $(2,1 \%)$ & 0 & $(0 \%)$ & \\
\hline Grado 4 & 2 & $(0,9 \%)$ & 0 & $(0 \%)$ & 0 & $(0 \%)$ & 2 & $(3,5 \%)$ & \\
\hline Perímetro abdominal (cm) & 109,5 & $5(14,0)$ & 102 & $(9,30)$ & 108,9 & $(10,63)$ & 120,28 & $(17,20)$ & $<0,001^{*}$ \\
\hline Saturación de oxígeno (\%) & 97,1 & $(1,36)$ & 97,47 & $(1,10)$ & 96,96 & $(1,27)$ & 96,80 & $(1,67)$ & $<0,01 *$ \\
\hline Frecuencia cardiaca (lat $/ \mathrm{min})$ & 75,9 & $(10,10)$ & 75,65 & $(8,29)$ & 76,15 & $(9,71)$ & 76,09 & $(12,73)$ & $0,94 *$ \\
\hline Obesidad & 113 & $(49,13 \%)$ & 34 & $(44,7 \%)$ & 48 & $(50,0 \%)$ & 31 & $(53,5 \%)$ & $<0,01 * *$ \\
\hline Obesidad mórbida & 34 & $(14,8 \%)$ & 6 & $(7,9 \%)$ & 11 & $(11,5 \%)$ & 17 & $(29,3 \%)$ & $<0,001^{* *}$ \\
\hline $\mathrm{IMC}\left(\mathrm{kg} / \mathrm{m}^{2}\right)$ & 33,3 & $(7,07)$ & 31,23 & $(4,78)$ & 32,35 & $(5,97)$ & 37,91 & $(9,10)$ & $<0,001$ \\
\hline
\end{tabular}

Tabla 4. Resumen de factores asociados a la presencia de riesgo de síndrome de apnea obstructiva del sueño utilizando tres reglas de predicción clínica

\begin{tabular}{|lccc|}
\hline Factores asociados & Escala de Berlín & Escala de Epworth & SACS \\
\hline Edad & - & - & - \\
Género & - & - & $\mathrm{X}$ \\
Horas de sueño & - & $\mathrm{X}$ & - \\
Mallampati & $\mathrm{X}$ & $\mathrm{X}$ & - \\
Hipertrofia amigdalina & $\mathrm{X}$ & $\mathrm{X}$ & - \\
Perímetro de cuello & $\mathrm{X}$ & $\mathrm{X}$ & $\mathrm{X}^{*}$ \\
\hline Perímetro abdominal & $\mathrm{X}$ & $\mathrm{X}$ & $\mathrm{X}$ \\
\hline Saturación $\mathrm{O}_{2}$ & - & $\mathrm{X}$ & $\mathrm{X}$ \\
IMC & $\mathrm{X}^{*}$ & $\mathrm{X}$ & $\mathrm{X}$ \\
\hline
\end{tabular}

SACS: Sleep apnea clinical score. IMC: Índice de Masa corporal. *Factor incluido en la escala. 


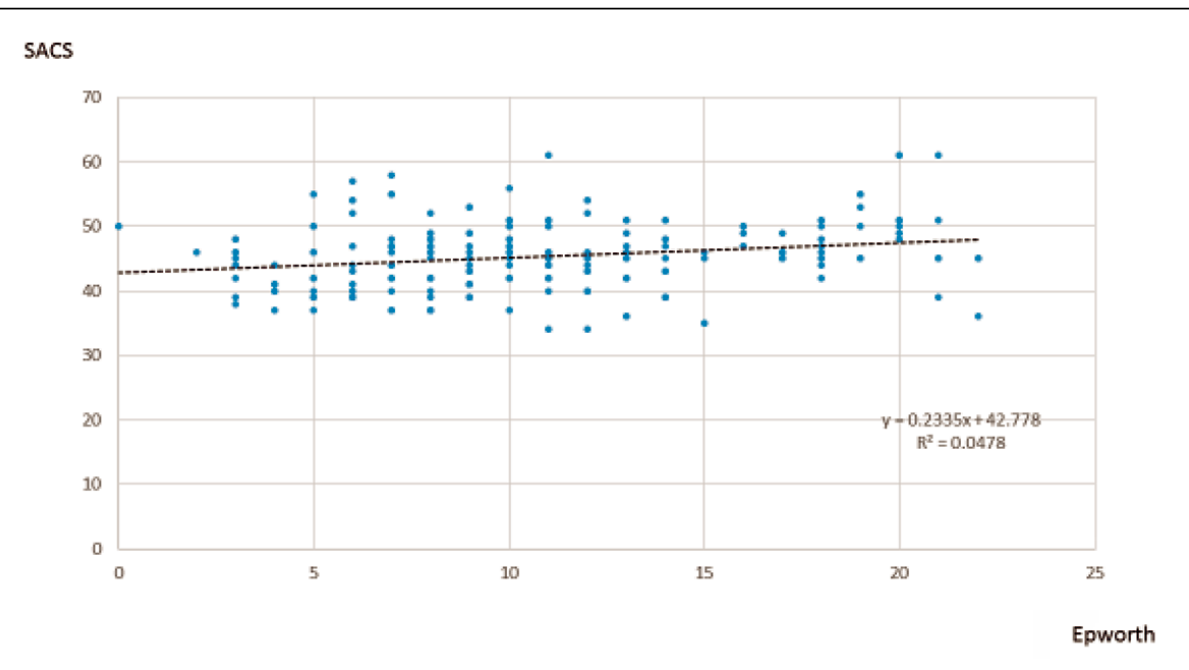

Figura 1. Correlación entre las escalas SACS y Epworth para la evaluación del riesgo de síndrome de apnea obstructiva del sueño. Lima, Perú. 2014

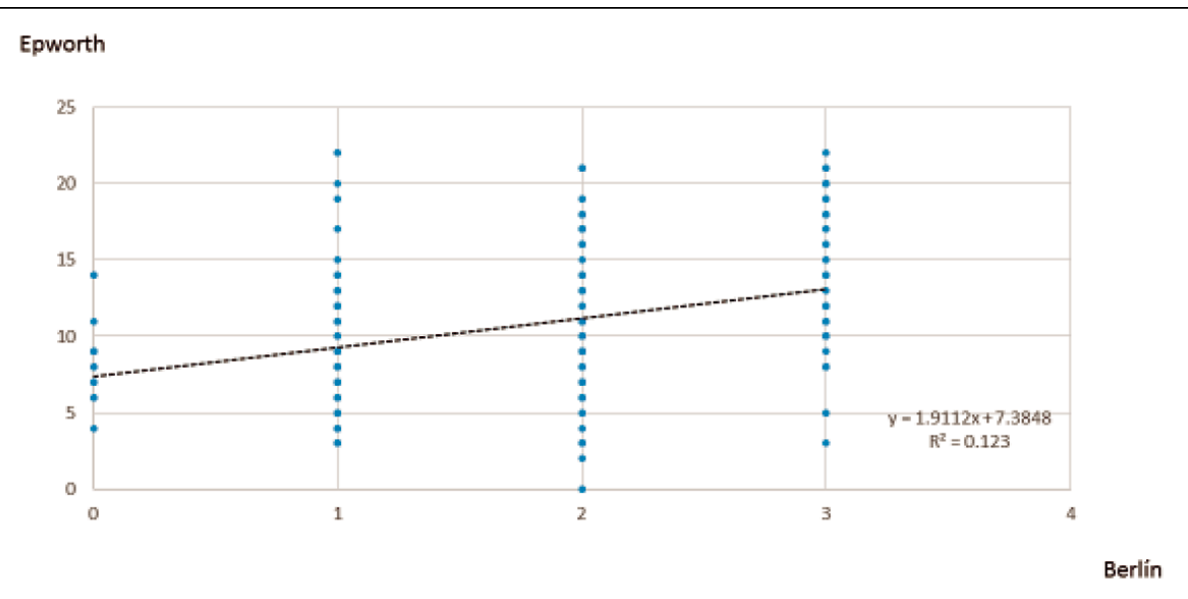

Figura 2. Correlación entre escalas de Epworth y Berlín para la valoración del riesgo de Síndrome de apnea obstructiva del sueño. Lima, Perú. 2014.

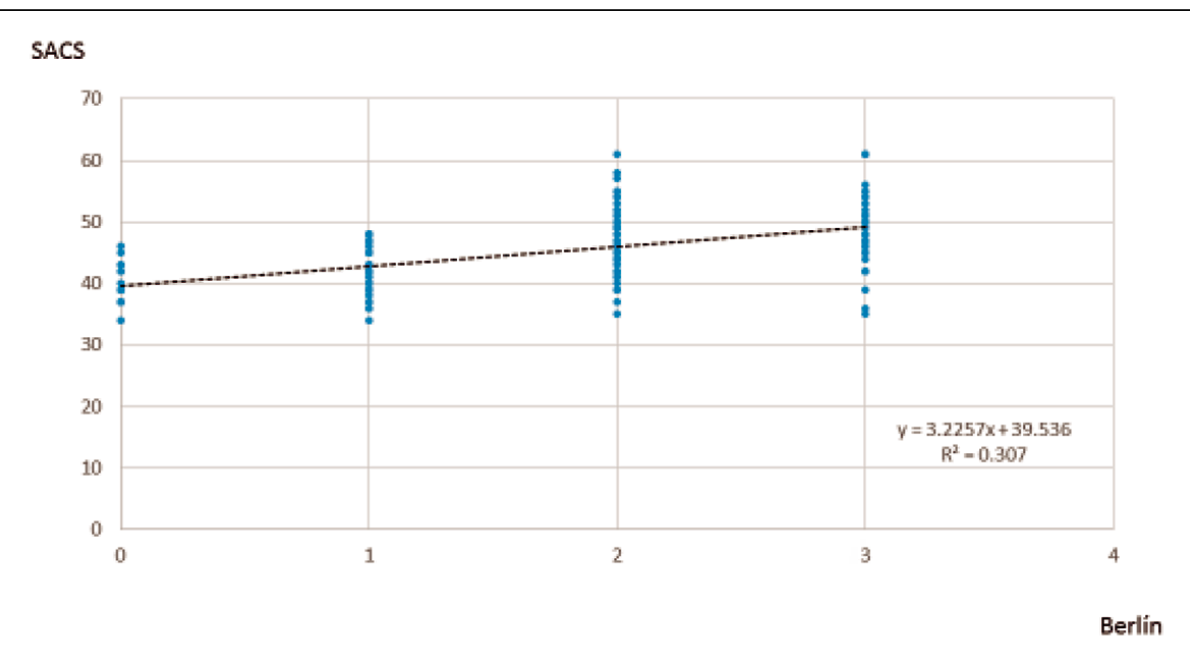

Figura 3. Correlación entre escalas SACS y Berlín para la valoración del riesgo de Síndrome de apnea obstructiva del sueño. Lima, Perú. 2014. 
buen marcador de obesidad central y predictor de $\mathrm{SAOS}^{15}$. Nuestro estudio encontró que el perímetro de cuello fue consistentemente asociado al riesgo de SAOS medido mediante los cuestionarios de Epworth y Berlín, lo que se correlaciona bien con resultados de otros estudios donde identificaron mayor riesgo para SAOS si dicho parámetro fue mayor de $40 \mathrm{~cm}$ en varones y 38 $\mathrm{cm}$ en mujeres ${ }^{5}$. El perímetro abdominal es otro buen marcador de obesidad. En nuestro estudio se encontró, de manera similar al perímetro de cuello, que la circunferencia abdominal fue otro de los parámetros consistentemente asociado al riesgo de SAOS. Estos resultados son similares a los descritos por Uribe y cols. ${ }^{23}$. Cabe resaltar que tanto el IMC, perímetro de cuello como la circunferencia abdominal son parámetros que pueden ser fácilmente evaluados en la práctica clínica por lo que su alteración en pacientes con ronquido habitual podría considerarse un criterio para la realización de un estudio de sueño.

Además de las asociaciones descritas, diversos estudios han mostrado que el SAOS se asocia a mala calidad de vida, accidentes vehiculares, accidentes laborales e incluso domésticos, síndromes depresivos y ansiedad; además de hipertensión arterial sistémica, cardiopatías isquémicas, arritmias y patología cerebrovascular ${ }^{24-27}$, por lo cual consideramos que los hallazgos de nuestro estudio implican la necesidad de realizar el despistaje de esta condición en todo paciente roncador que acuda a la consulta médica.

Entre las limitaciones del estudio, debemos mencionar que el porcentaje de pérdidas de participantes por fichas de recolección de datos incompletas fue de $3,7 \%$, que representa una pequeña cifra y no consideramos que invalide los resultados del estudio. Por otro lado, nuestros hallazgos al ser procedentes de un solo centro de referencia no son generalizables a la población general o a pacientes atendidos en hospitales o centros de salud de menores niveles de atención. Por otro lado, el tamaño muestral podría no ser suficiente para la evaluación de asociaciones de magnitud relativamente menor. Otra limitación puede ser el no contar con la descripción detallada de las condiciones clínicas motivo de consulta en adición al ronquido. Finalmente, al no ser un estudio ciego, puede existir el riesgo de sesgo en las mediciones y resultados de los cuestionarios. Pese a las limitaciones descritas, consideramos que nuestro estudio brinda información valiosa que evidencia la magnitud del problema de riesgo de SAOS entre roncadores habituales.

Aquellos pacientes que presentan moderado $\mathrm{y}$ alto riesgo de SAOS deberían ser sometidos a pruebas confirmatorias, idealmente la polisomnografía, pero también se pueden realizar estudios simplificados con polígrafos respiratorios los que han sido validados como herramienta diagnóstica y para la titulación de CPAP en pacientes con alto riesgo de SAOS o en casos de escenarios de recursos limitados incluso oxímetros nocturnos (pese a que su utilidad para el manejo de SAOS no ha sido demostrada) $)^{28-31}$. Los estudios simplificados, utilizados en los pacientes apropiados, pueden permitir priorizar la realización de la polisomnografía en quienes exista duda diagnóstica. Finalmente consideramos necesario realizar estudios en el Perú y Latinoamérica que evalúen la capacidad diagnóstica de las escalas SACS, Epworth y Berlín utilizando la polisomnografía como estándar de referencia, para determinar cuál de ellas podría ser aplicada como mejor sistema de tamizaje inicial para la pesquisa de SAOS en pacientes roncadores. La elección de la mejor prueba de tamizaje es especialmente importante dado el alto costo y dificultad para realización de estudios polisommnográficos.

\section{Bibliografía}

1.- AMERICAN ACADEMY OF SLEEP MEDICINE. International classification of sleep disorders. Diagnostic and coding manual. $2^{\text {nd }} \mathrm{ed}$. Westchester, Illinois: American Academy of Sleep Medicine 2012; 51.

2.- LEGER D, POURSAIN B, NEUBAUER D, UCHIYAMA M. An international survey of sleeping problems in the general population. Curr Med Res Opin 2008; 24 : 307-17.

3.- YOUNG T, PEPPARD PE, GOTTLIEB DJ. Epidemiology of obstructive sleep apnea: a population health perspective. Am J Respir Crit Care Med 2002; 165 : 1217-39.

4.- YOUNG T, PALTA M, DEMPSEY J, SKATRUD J, WEBER S, BADR S. The occurrence of sleep-disordered breathing among middle-aged adults. N Engl J Med 1993; 328: 1230-5.

5.- HIESTAND D, BRITZ P, GOLDMAN M, PHILLIPS B. Prevalence of symptoms and risk of sleep apnea in the US population: results from the national sleep foundation sleep in America 2005 Poll. Chest 2006; 130: 780-6.

6.- TORRE-BOUSCOULET L, VÁZQUEZ-GARCÍA JC, MUIÑO A, MARQUEZ M, LOPEZ MV, DE OCA MM, et al. Prevalence of sleep-related symptoms in four Latin American cities. J Clin Sleep Med 2008; 4: 579-85.

7.- BASSETTI CL, MILANOVA M, GUGGER M. Sleep disordered breathing and acute ischemic stroke. Stroke 2006; 37: 967-72.

8.- YAGGI HK, CONCATO J, KERNAN WN, LICHT- 
MAN JH, BRASS LM, MOHSENIN V. Obstructive sleep apnea as a risk factor for stroke and death. N Engl J Med 2005; 353: 2034-41.

9.- HAPONIK EF, SMITH PL, MEYERS DA, BLEECKER ER. Evaluation of sleep-disordered breathing. Is polysomnography necessary? Am J Med 1984; 77: 671-7.

10.- JOHNS MW. Sensitivity and specificity of the multiple sleep latency test (MSLT), the maintenance of wakefulness test and the Epworth sleepiness scale: failure of the MSLT as a gold standard. J Sleep Res 2000; 9: 5-11.

11.- ROSENTHAL LD, DOLAN DC. The Epworth sleepiness scale in the identification of obstructive sleep apnea. J Nerv Ment Dis 2008; 196: 429-31.

12.- FLEMONS WW. Clinical practice. Obstructive sleep apnea. N Engl J Med 2002; 347: 498-504.

13.- NETZER NC, STOOHS RA, NETZER CM, CLARK K, STROHL KP. Using the Berlin Questionnaire to identify patients at risk for the sleep apnea syndrome. Ann Intern Med 1999; 131: 485-91.

14.- SAGASPE P, LEGER D, TAILLARD J, BAYON V, CHAUMET G, PHILIP P. Might the Berlin Sleep Questionnaire applied to bed partners be used to screen sleep apneic patients? Sleep Medicine 2010; 11: 479-83.

15.- JOHNS MW. A new method for measuring daytime sleepiness: the Epworth Sleepiness Scale. Sleep 1991; 14: $540-5$

16.- MILLER JN, BERGER AM. Screening and assessment for obstructive sleep apnea in primary care. Sleep Med Rev 2016;29:41-51.

17.- ROSALES E. Estudio de validez y confiabilidad de la escala de somnolencia de Epworth en población peruana y modificación de la escala para población que no conduce vehículos motorizados [Tesis de Maestría]. Lima: Facultad de Medicina, Universidad Peruana Cayetano Heredia; 2009.

18.- POLANÍA-DUSSAN, I. ESCOBAR-CÓRDOBA F, ESLAVA-SCHMALBACH J, NETZER N. Validación colombiana del cuestionario de Berlín. Revista de la Facultad de Medicina; 2014; 61: 231-8.

19.- ANDRADE MA, CASCANTE JA, IRIDOY A, HERNÁNDEZ M, CEBOLLERO P, EGUÍA VM, et al. Modified sleep apnea clinical score: Is useful in our clinical practice? Eur Respir J 2011; 38: 2201.

20.- HASSAN I. Comparison of four sleep questionnaires for screening obstructive sleep apnea. Egypt J Chest Dis Tuberc 2012; 61: 433-41.

21.- YE L, PIEN GW, WEAVER TE. Gender differences in the clinical manifestation of obstructive sleep apnea Sleep Med 2009; 10: 1075-84.
22.- PALLA A, DiGIORGIO M, CARPENE N, ROSSI G, D'AMICO I, SANTINI F, et al. Sleep apnea in morbidly obese patients: prevalence and clinical predictivity. Respiration 2009; 78: 134-40.

23.- URIBE-ECHEVARRIA E, ALVAREZ D, GIOBELLINA R, URIBE-ECHEVARRIA E. Valor de la Escala de Somnolencia de Epworth en el diagnóstico del síndrome de Apneas Obstructivas del Sueño. Medicina 2000; 60: 902-6.

24.- NARKIEWICZ K, MONTANO N, CUGLIATI C, VAN DE BORNE PJ, DYKEN ME, SOMERS VK. Altered cardiovascular variability in obstructive sleep apnea. Circulation 1998; 98: 1071-7.

25.- SMITH LA, CHONG DW, VENELLE M, DENVIR MA, NEWBY DE, DOUGLAS NJ. Diagnosis of sleepdisordered breathing in patients with chronic heart failure: evaluation of a portable limited study system. J Sleep Res 2007; 16: 428-35.

26.- MEZZANOTTE WS, TANGEL DJ, WHITE DP. Waking genioglossal electromyogram in sleep apnea patients versus normal controls (a neuromuscular compensatory mechanism). J Clin Invest 1992; 89 1571-9.

27.- COLLOP NA, ANDERSON WM, BOEHLECKE B, CLAMAN D, GOLDBERG R, GOTTLIEB DJ, et al Clinical guidelines for the use of unattended portable monitors in the diagnosis of obstructive sleep apnea in adult patients. Portable Monitoring Task Force of the American Academy of Sleep Medicine. J Clin Sleep Med 2007; 3: 737-47.

28.- BRIDEVAUX PO, FITTING JW, FELLRATH JM, AUBERT JD. Inter-observer agreement on apnoea hypopnoea index using portable monitoring of respiratory parameters. Swiss Med Wkly 2007; 137: 602-7.

29.- TORRE-BOUSCOULET L, CASTORENA-MALDONADO A, BAÑOS-FLORES R, VÁZQUEZ-GARCÍA JC, MEZA-VARGAS MS, PÉREZ-PADILLA R. Agreement between oxygen desaturation index and apneahypopnea index in adults with suspected obstructive sleep apnea at an altitude of $2240 \mathrm{~m}$. Arch Bronconeumol 2007; 43: 649-54.

30.- HEINZER RC, STANCHINA ML, MALHOTRA A, FOGEL RB, PATEL SR, JORDAN AS, et al. Lung volume and continuous positive airway pressure requirements in obstructive sleep apnea. Am J Respir Crit Care Med 2005; 172: 114-7.

31.- MULGREW AT, FOX N, AYAS NT, RYAN CF. Diagnosis and initial management of obstructive sleep apnea without polysomnography: a randomized validation study. Ann Intern Med 2007;146:157-66.
Correspondencia a:

Dr. Alonso Soto Tarazona

Hospital Nacional Hipólito Unánue. Lima, Perú

Email: sotosolari@yahoo.com 